\title{
Whistler intensities above thunderstorms
}

\author{
J. Fiser ${ }^{1}$, J. Chum ${ }^{1}$, G. Diendorfer ${ }^{2}$, M. Parrot ${ }^{3}$, and O. Santolik ${ }^{1,4}$ \\ ${ }^{1}$ Institute Of Atmospheric Physics, Bocni II/1401, 14131 Prague, Czech Republic \\ ${ }^{2}$ Austrian Electrotechnical Association (OVE-ALDIS), Kahlenberger Str. 2A, 1190 Vienna, Austria \\ ${ }^{3}$ LPCE/CNRS, 3A Avenue de la Recherche Scientifique, 45071 Orléans, France \\ ${ }^{4}$ Charles University, Faculty of Mathematics and Physics, V Holesovickach 2, 18000 Prague, Czech Republic
}

Received: 27 February 2009 - Revised: 13 November 2009 - Accepted: 14 December 2009 - Published: 13 January 2010

\begin{abstract}
We report a study of penetration of the VLF electromagnetic waves induced by lightning to the ionosphere. We compare the fractional hop whistlers recorded by the ICE experiment onboard the DEMETER satellite with lightning detected by the EUCLID detection network. To identify the fractional hop whistlers, we have developed software for automatic detection of the fractional-hop whistlers in the VLF spectrograms. This software provides the detection times of the fractional hop whistlers and the average amplitudes of these whistlers. Matching the lightning and whistler data, we find the pairs of causative lightning and corresponding whistler. Processing data from 200 DEMETER passes over the European region we obtain a map of mean amplitudes of whistler electric field as a function of latitudinal and longitudinal difference between the location of the causative lightning and satellite magnetic footprint. We find that mean whistler amplitude monotonically decreases with horizontal distance up to $\sim 1000 \mathrm{~km}$ from the lightning source. At larger distances, the mean whistler amplitude usually merges into the background noise and the whistlers become undetectable. The maximum of whistler intensities is shifted from the satellite magnetic footprint $\sim 1^{\circ}$ owing to the oblique propagation. The average amplitude of whistlers increases with the lightning current. At nighttime (late evening), the average amplitude of whistlers is about three times higher than during the daytime (late morning) for the same lightning current.
\end{abstract}

Keywords. Ionosphere (Wave propagation) - Meteorology and atmospheric dynamics (Lightning) - Radio science (Waves in plasma)

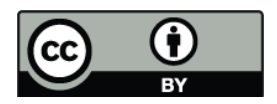

Correspondence to: J. Chum

(jachu@ufa.cas.cz)

\section{Introduction}

Electron whistlers are electromagnetic waves propagating in magnetized plasmas at frequencies below the local electron cyclotron frequency $f_{\mathrm{ce}}$ and plasma frequency $f_{\mathrm{p}}$, and above the ion cyclotron frequencies. After the early works of Barkhausen (1930) and Eckersley (1935), Storey (1953) gave their correct physical explanation and showed that ducted whistlers originate in impulsive atmospherics (ultra-wide band electromagnetic signal produced by lightning) propagating through the ionosphere and following the magnetic field lines. During their journey they become dispersed as a consequence of dispersive properties of the ionized medium in the magnetosphere. Analyzing the dispersion of whistlers, Storey (1953) showed that the plasma densities at the equatorial plane at the distances of several Earth radii are much larger than was previously supposed. He also showed that the whistlers observed at different locations are correlated at distances up to $\sim 1000 \mathrm{~km}$. The same result was also confirmed by Crary et al. (1956).

Helliwell (1965) used whistler dispersions to determine the electron densities in the inner magnetosphere. He also summarized the characteristics of whistlers in the Earth's magnetosphere and the foregoing research on this phenomenon. In general the whistlers can propagate at oblique angles with respect to magnetic field lines and are refracted by the combined effects of the Earth's plasmasphere and magnetosphere (Kimura, 1966; Shklyar et al., 2004). Whistler mode waves still attract attention because they can interact with energetic electrons and cause their pitch angle scattering leading to precipitation (Kennel and Petschek, 1966; Brinca, 1972; Abel and Thorne, 1998a,b; Bortnik et al., 2003) or acceleration (Trakhtengerts et al., 2003).

Lightning, sources of whistlers, is divided into two basic types - cloud-to-ground (CG) and in-cloud discharges which includes cloud-to-cloud, intracloud and cloud-to-sky discharges. In-cloud discharges occur more frequently than

Published by Copernicus Publications on behalf of the European Geosciences Union. 
$\mathrm{CG}$ discharges. The in-cloud to $\mathrm{CG}$ occurrence ratio at $\sim 50^{\circ}$ latitude computed from an empirical relationship derived by Prentice and Mackerras (1977) is 2.3:1. The intensities of incloud lightning are relatively weaker than those of CG lightning (Betz et al., 2009). As for lightning used in our study, mean values of absolute peak current are $12.5 \mathrm{kA}$ and $19 \mathrm{kA}$ for in-cloud and CG discharges, respectively. Maxima of occurrence histograms of lightning absolute peak currents are at $4 \mathrm{kA}$ and $9 \mathrm{kA}$ for in-cloud and CG discharges, respectively. The lightning detection systems are constructed to be mainly sensitive to CG discharges because of their hazardous potential on men, buildings, crops etc. As we mentioned previously, lightning are sources of impulsive atmospherics. These propagate in a waveguide formed by the Earth's surface and lower boundary of the ionosphere. Some of their energy can leak into the ionosphere, where it starts to propagate approximately along the magnetic field lines in the whistler wave mode (Helliwell, 1965). In-situ rocket experiments showed that this energy can leak into the ionosphere at distances as far as $1000 \mathrm{~km}$ from the lightning (Holzworth et al., 1999). The same result was also confirmed by spacecraft measurements (Chum et al., 2006; Santolik et al., 2009).

The aim of our study is to provide better information about the area over which the lightning energy enters the ionosphere and about typical whistler intensities above the thunderstorm regions. Therefore, this study is concerned with whistlers which have not crossed the magnetic equatorial plane. These whistlers are usually called fractional hop whistlers or 0+ whistlers (Smith and Angerami, 1968). First results have been presented by Chum et al. (2006) who showed that the width of the area of penetration is $\sim 2000 \mathrm{~km}$ and that it is possible to find the causative lightning to whistlers observed at satellites orbiting at different altitudes. Because this study was based on relatively low number of data owing to the slow manual detection of whistlers in the spectrograms, it provided only a rough estimate of the size of the penetration area and could not compare the average amplitudes of whistlers at night with those observed during the day. Therefore, we have developed a method for automatic detection of the fractional hop whistlers. This allowed us to make an analysis based on a larger amount of data. The results of this new, more detailed analysis are presented in this paper.

\section{Experimental data}

The lightning data were provided by the European Cooperation for Lightning Detection (EUCLID, www.euclid.org). The fractional-hop whistlers were detected using the ICE experiment (Instrument Champ Electrique = Electric Field Instrument) onboard the French satellite DEMETER (Detection of Electro-Magnetic Emissions Transmitted from Earthquake Regions). The basic description of the DEMETER mission can be found in Cussac et al. (2006) and Lagoutte et al. (2006).

\subsection{The lightning detection network EUCLID}

The EUCLID network is a collaboration among national lightning detection networks with the aim to identify and detect lightning all over the European area. This network has been in operation since 2000 and consists of 135 sensors (2008). The sensors detect electromagnetic signals emitted by lightning return strokes. After GPS time-stamping, these data are processed by a central analyzer which calculates the location of the lightning (given in geographical coordinates), its inferred peak current including polarity, and type (CG/incloud). The accuracy of the determination of lightning position is $\sim 1 \mathrm{~km}$ and the time of detection is given in millisecond precision. The network facilities are designed to provide best performance in the detection of CG lightning. Only a few percent of all in-cloud lightning are detected and the detection efficiency of in-cloud lightning is not normalized over the network area (Chum et al., 2006).

\subsection{DEMETER satellite, VLF data}

The DEMETER satellite was launched into a quasi sunsynchronous orbit at the altitude of $710 \mathrm{~km}$ in June 2004 . At the end of 2005, this orbit was lowered to $660 \mathrm{~km}$. In our study, VLF data from the ICE experiment are used. The primary objective of this experiment, as of most of the experiments on the satellite, is a detection of electromagnetic signatures associated with seismic activity. A secondary objective of the experiment is to investigate the natural and artificial electromagnetic waves in the plasma environment of the Earth, including whistlers (Berthelier et al., 2006).

The ICE experiment consists of 4 sensors formed by spherical aluminium electrodes with embedded preamplifiers, mounted on the ends of $4 \mathrm{~m}$ long booms, along with associated electronics. Measuring a potential difference between two sensors, a component of the electric field along the axis defined by these two sensors is provided. The frequency range of this experiment is from DC to $3.175 \mathrm{MHz}$. It is divided into four frequency channels (DC/ULF, ELF, VLF and HF). In the VLF channel $(15 \mathrm{~Hz}-17.4 \mathrm{kHz})$, which we are interested in, only one component of the electric field is available. In the nominal configuration, the field component along the axis perpendicular to the orbital plane is measured, but one of two other components can be selected by a telecommand (Berthelier et al., 2006).

The satellite operates in two modes. The survey mode is used all around the Earth. Averaged data are collected in this mode; onboard processing is performed to reduce the telemetry flow. In the VLF range, data are typically averaged over $0.512 \mathrm{~s}$ (Berthelier et al., 2006). In the burst mode, which is activated above seismically active regions, including most of the area covered by the EUCLID network, data are recorded 
with a higher sample rate. Because the survey mode has insufficient time resolution, we have used the burst mode data for this study. In this mode, the waveform is recorded with the sampling frequency of $40 \mathrm{kHz}$ in the VLF channel. Besides the time of recording we also use the location of the satellite on the orbit and the position of the magnetic footprints in our analysis. The magnetic footprints are obtained by projecting the satellite location along the magnetic field lines to the altitude of $110 \mathrm{~km}$, where the lower boundary of the ionosphere is assumed. The altitude of $110 \mathrm{~km}$ was chosen mainly from practical reasons because the coordinates of the magnetic footprints at this altitudes are provided by the DEMETER data centre and they are added into the data files. The real lower boundary of the ionosphere is lower at about $80 \mathrm{~km}$. However, considering $\sim 65^{\circ}$ inclination of the magnetic field in the Central Europe, we get $\sim 12 \mathrm{~km}$ horizontal difference between the magnetic field lines at $80 \mathrm{~km}$ and $110 \mathrm{~km}$. This horizontal difference is much smaller than the $100 \mathrm{~km}$ horizontal resolution used in our statistical analysis (see Sect. 3). Therefore, we take the magnetic footprints at $110 \mathrm{~km}$ that are already provided. Moreover, the transformation of free space electromagnetic wave mode into the whistler wave mode will probably take place over some range of altitudes. So it may be reasonable to consider the start of pure whistler mode propagation higher than at $80 \mathrm{~km}$. Note that the wavelength of $3 \mathrm{kHz}$ waves is $100 \mathrm{~km}$ in free space and $\sim 10 \mathrm{~km}-1 \mathrm{~km}$ for whistler mode waves propagating in the lower ionosphere, depending on the plasma density and propagation angle. The analysis of the wave mode conversion is beyond the scope of the present experimental paper.

Owing to its orbit, the DEMETER satellite regularly passes over about the same location two times a day. It flies northward approximately between 8 a.m. and 10 a.m. (LT), whereas it moves southward in the evening hours, approximately between 8 p.m. and 10 p.m. (LT). Thus, we can compare daytime (late morning) observations with those recorded during nighttime (late evening).

We do not expect any substantial differences in the received amplitudes of the electric field fluctuations that would be caused by the difference in the direction of the receiving antenna during the nightside and dayside half-orbits. The whistler waves are expected to be approximately circularly polarized perpendicular to the local magnetic field line. The magnetic declination is small in Europe, and the magnetic field lines are inclined $\sim 65$ degrees from the horizontal plane. The inclination of the orbit causes therefore only small differences (less than several degrees) in the angle between the antenna and the polarization plane of the waves between the nightside and dayside half-orbits.

\subsection{Data processing}

A determination of whistler times is based on the crosscorrelation of a reference spectrogram containing a reference whistler with the VLF spectrograms in the frequency range

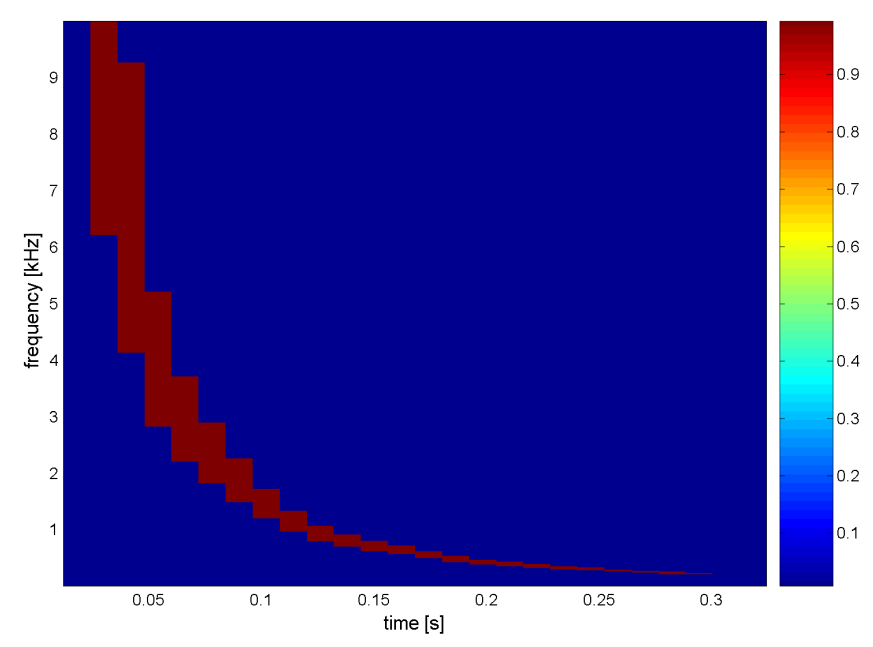

Fig. 1. Normalized reference whistler used for daytime cases. See the text for more details.

$0-10 \mathrm{kHz}$. The spectrograms are computed from the waveforms using an FFT algorithm with overlapping time windows. The reference whistler has been constructed by a superposition of several distinct fractional hop whistlers found visually in the spectrograms. Because the whistler dispersion depends on the plasma density, it is different in the morning and in the evening. Therefore we have defined two reference whistlers, one for late morning and one for late evening.

The noise background was artificially removed from the reference spectrogram. This was done by first removing from the spectrogram all the pixels whose spectral intensity was below a threshold. The threshold was set in such a way so that all the pixels remaining would belong to the whistler and not to other types of emissions. These pixels form $\left(t_{i}, f_{i}\right)$ pairs that define the whistler trace in the frequency - time plane. We have also found a frequency width of the whistler trace for each time interval in which we computed spectrum. Note that the frequency resolution is much better than the frequency width of the whistler trace in these time intervals; thus, we have much more $\left(t_{i}, f_{i}\right)$ pairs than time intervals. Note also that some pixels that belong to the whistler can be missing in the $\left(t_{i}, f_{i}\right)$ pairs selected by this procedure. Therefore, we found a best regression for these pairs using the Least Squares (LS) method and a polynomial of 9-th order. We also attempted to fit the $\left(t_{i}, f_{i}\right)$ pairs using the formula $t=D f^{-\frac{1}{2}}$, but the match was worse, especially at the lowest frequencies. Having found the regression curve, we returned to the original spectrogram containing the superposed intense whistlers and set all the pixels which were more distant from the regression curve than one half of the frequency width to zero. This reference whistler has a non-uniform amplitude. We therefore defined a normalized whistler by setting all the nonzero pixels to unit amplitude (see Fig. 1). 


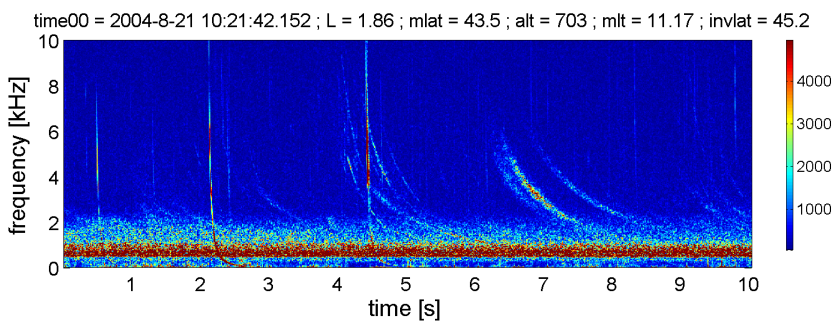

cross-correlation with a reference whistler

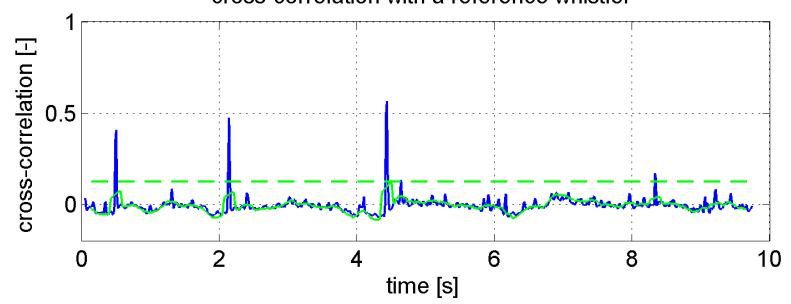

Fig. 2. Top: An example of the spectrogram computed from the ICE burst mode data. Bottom: The cross-correlation function (blue line) of the spectrogram with the reference whistler, 13-point running average of the cross-correlation function (solid green line) and 0.125 detection threshold (dashed green line). See the text for more details.

We compute two-dimensional cross-correlation function $c(t)$ defined by Eq. (1).

$$
c(t)=\frac{\sum_{i=1}^{n} \sum_{j=1}^{m}\left(a_{i, j+t}-\langle a\rangle_{t}\right)\left(b_{i, j}-\langle b\rangle\right)}{\sqrt{\sum_{i=1}^{n} \sum_{j=1}^{m}\left(a_{i, j+t}-\langle a\rangle_{t}\right)^{2} \sum_{i=1}^{n} \sum_{j=1}^{m}\left(b_{i, j}-\langle b\rangle\right)^{2}}}
$$

where $\langle a\rangle_{t}$ is the mean spectral amplitude of the spectrogram which begins at time $t$ and has the same size as the normalized reference spectrogram, $a_{i, j+t}$ is the spectral amplitude at frequency $f_{i}$ and time $t+j \times d t$ of the same part of the VLF spectrogram ( $d t$ is the time resolution of the spectrogram, $d t \cong 12 \mathrm{~ms}) .\langle b\rangle$ is the mean spectral amplitude of the normalized reference spectrogram, $b_{i, j}$ is the amplitude spectral intensity of the normalized reference spectrogram, $n$ is the number of frequency bins in the spectrogram and $m$ is the number of time intervals in the spectrogram. Since we have often observed relatively intense natural emissions having noise character in the frequency range $\sim 0.4-\sim 2 \mathrm{kHz}$, see e.g. Fig. 2, we have excluded these frequencies from the analysis when calculating $c(t)$. Figure 2 also shows an example of the cross-correlation function $c(t)$.

Obviously, a fractional-hop whistler $(0+$ whistler $)$ in the spectrogram produces a relatively distinct peak in the crosscorrelation function. By measuring the positions of these peaks in time, we can determine times $\left(t_{w}\right)_{i}$ at which whistlers occurred. In other words, we detect whistlers at times where the peaks of the cross-correlation function occur.
We take only those peaks which are higher than a threshold $p=k \times\langle c(t)\rangle$, where $\langle c(t)\rangle$ is a 13-point running average of $c(t)$. At the same time we require $p>r$. Coefficients $k=1.4$ and $r=0.125$ were chosen experimentally to avoid false detection, but to detect intense whistlers. Whistlers with intensities close to the background noise may be missed. Note that all the intense 0+ whistlers in Fig. 2 (upper plot) are detected and that the whistlers propagating from the other hemisphere (with higher dispersion) observed, e.g. at time $\sim 7 \mathrm{~s}$ do not result in the peaks of the cross-correlation function in Fig. 2 (lower plot).

We have compared results of detection while using a reference whistler (spectrogram) and a normalized reference whistler. The obtained results were very similar; nevertheless, the cross-correlation with the normalized reference whistler (spectrogram) gave about 5\% higher efficiency in detecting whistlers. Therefore, in what follows, we present the results obtained by the detection based on the normalized reference whistler. The reason that we detected more whistlers using the normalized reference whistler compared to the procedure based on the unnormalized reference whistler can be the low number of whistlers used to create the particular reference whistler. It was relatively time demanding, especially for nighttime cases, to find a "solitary" distinct whistler without the presence of other emissions in the spectrograms. Therefore, several intense whistlers determine the spectral intensity distribution of the reference whistler. If that spectral intensity doesn't match the most frequent spectral intensity distribution of the observed whistlers or if their spectral intensity varies from case to case, then the maxima of cross-correlation functions become smaller and less whistlers are detected. So, the normalized reference whistler with the uniform spectral intensity distribution can give better results. We stress that the difference between the number of detected whistlers obtained by these two approaches was small, around 5\%.

In Table 1 we show the comparison between the results of the described automatic method and of the visual method used in the initial study by Chum et al. (2006). The automatic method detects more whistlers compared to the visual selection. We should note that manual detection by visual inspection is a subjective matter. First, only distinct whistlers were included into the list of detected whistlers. Second, different persons could use a different subjective "threshold" to decide which whistlers to include in the list and which to reject.

Mean amplitudes of the detected whistlers are computed by summing their power spectral intensities in the frequency range from $\sim 300 \mathrm{~Hz}$ to $10 \mathrm{kHz}$ and averaging over the whistler duration. Only those pixels in the spectrogram which correspond to the non-zero pixels forming the reference whistler enter into these calculations performed only at the times of detections of whistlers. We make a list of all the times at which whistlers were detected on the satellite and of their mean amplitudes. 
Table 1. Number of whistlers detected by automatic method and by manual method.

\begin{tabular}{cccc}
\hline Date & Time & Automatic method & Visual method \\
\hline 14 Sep 2004 & 10:19:02-10:25:02 & 598 & 512 \\
16 Jun 2005 & 20:54:02-20:58:58 & 790 & 391 \\
29 Jun 2005 & 22:01:02-22:04:30 & 873 & 259 \\
\hline
\end{tabular}

The whistler times $\left(t_{\mathrm{w}}\right)_{i}$ are compared with the lightning times $\left(t_{1}\right)_{j}$ obtained from the EUCLID network in the same time interval and a matrix $\left\{t_{\mathrm{wl}}\right\}_{i j}=\left(t_{w}\right)_{\mathrm{i}}-\left(t_{1}\right)_{j}$ of all possible time differences between detected whistlers and lightning is created. Thus, $\left(t_{\mathrm{wl}}\right)_{i j}$ is the time difference between the detection of $i$-th whistler and $j$-th lightning. We use a statistical approach. First, we find the most probable time difference $t_{\mathrm{wl}}$ between whistlers and their causative lightning by the method described by Chum et al. (2006). This time difference $t_{\mathrm{wl}}$ corresponds to the peak in the occurrence histogram for all the possible time differences between the times of whistler and lightning detections for a given satellite pass. We assume that this time difference equals propagation time of the waves from the discharges to the satellite plus a possible small time shift between clocks used in the EUCLID network and on the DEMETER satellite. The time difference is assumed to be constant during each analyzed time interval. Next, we select from all possible lightning-whistler pairs those, which satisfy the following relation:

$\left(t_{\mathrm{wl}}-0.0125 \mathrm{~s}<\left(t_{\mathrm{wl}}\right)_{i j}<t_{\mathrm{wl}}+0.0125 \mathrm{~s}\right)$

Note that $0.0125 \mathrm{~s}$ is approximately the time resolution of the spectrograms. We suppose that whistlers and lightning that satisfy the above mentioned relation correspond to whistlers and their causative lightning. We also estimate the position of the maximum of histogram with an accuracy better than $0.0125 \mathrm{~s}$. To do this estimation, we find a maximum of polynomial of the second order interpolated through the histogram maximum and two adjacent points. This maximum occurs at time $t_{\mathrm{wl}}^{*}$. In the case that more than one lightning satisfies the relation (2) for a given whistler, we choose that lightning whose absolute difference $\left|\left(t_{\mathrm{wl}}\right)_{i j}-t_{\mathrm{wl}}^{*}\right|$ is smaller.

\section{Obtained results and discussion}

We have analyzed data from 186 satellite passes over the European region during the 3 year period from September 2004 to September 2007. These time intervals (satellite passes) have been selected in days with significant storm activity; 97 of them were recorded during the daytime (late morning) and 89 at nighttime (late evening). Figure 3 shows one satellite pass and positions of lightning discharges assigned to whistlers during this pass (blue circles $=-\mathrm{CG}$, green stars $=$ in-cloud). The two lines in the plot represent different projections of the satellite position. The purple line indicates

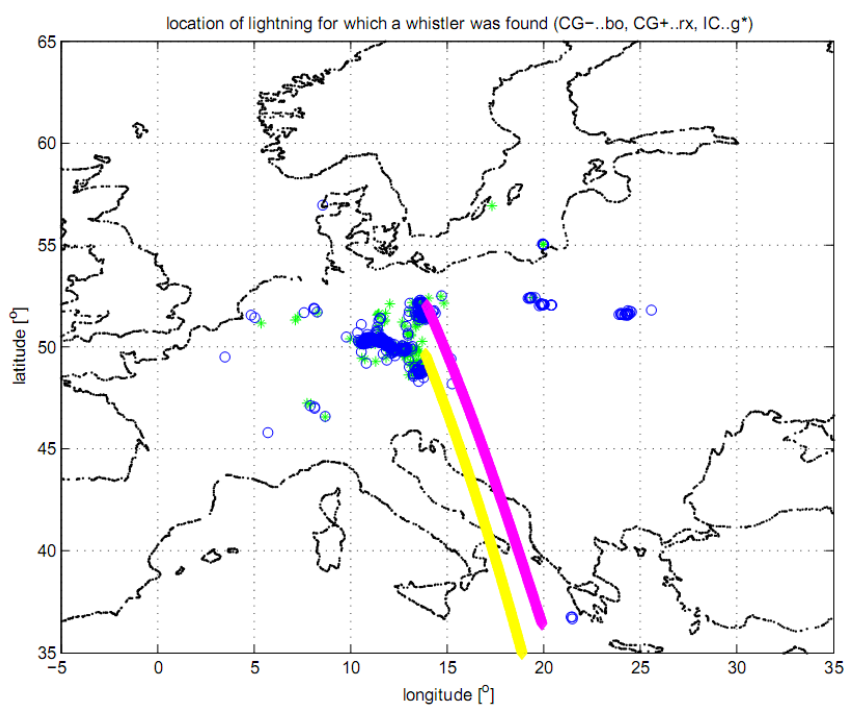

Fig. 3. An example of lightning activity during one pass of the satellite over the European region (evening pass on 29 July 2005).

the magnetic footprints, whereas the yellow line is the vertical projection to the ground. The positions of the satellite are displayed only in the time interval of the burst mode data recording. In our analysis, we have found 30000 lightningwhistler pairs. Because of the low sensitivity of the detection network to in-cloud lightning, most of the lightning assigned to the whistlers are $\mathrm{CG}$ discharges (ratio of the assigned incloud to the assigned $\mathrm{CG}$ discharges is $\sim 0.25$ ). For these causative lightning-whistler pairs, the distance between the lightning locations and the magnetic footprints can be found. Subsequently, we can analyze the average whistler amplitude as a function of the lightning current and distance from the magnetic footprint.

The plots presented on the left hand side in Fig. 4 and Fig. 5 show the number of all lightning detected by the EUCLID network (top) and the ratio of the lightning assigned to whistlers to all lightning detected by the EUCLID network (bottom) as a function of the latitudinal and longitudinal distance from the magnetic footprint. The data are organized into bins with resolution of $1^{\circ}$ in latitude and longitude. Positive values of the difference in latitude or longitude, respectively, mean that lightning occurred to the south or to the west of the magnetic footprint. White bins in the bottom 

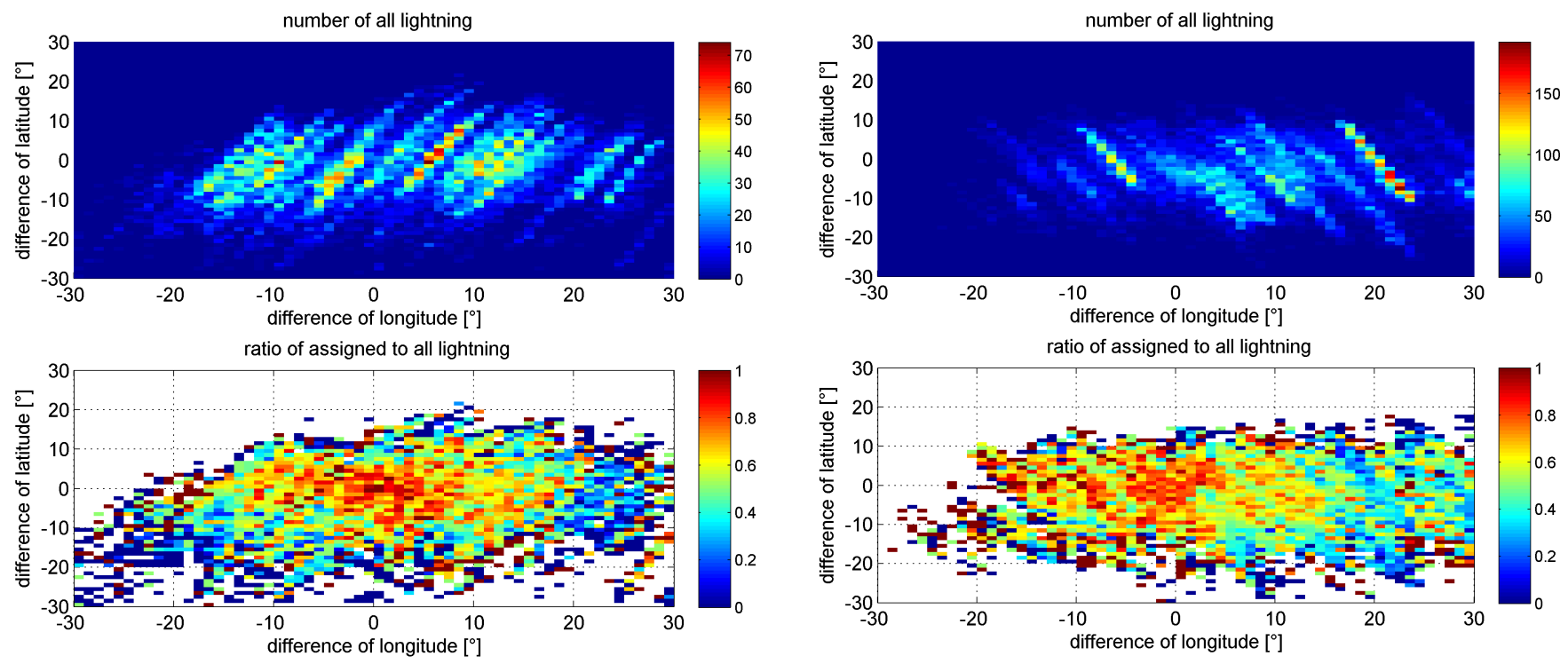

Fig. 4. Top: number of all lightning detected by the EUCLID network as a function of longitudinal and latitudinal difference between the magnetic footprint and locations of lightning. Bottom: Ratio of the assigned to all lightning (daytime cases).

plots marks the intervals with zero number of data. In general, the ratio of the assigned to all lightning is highest for lightning which are close to the magnetic footprint location and decreases with distance. At large distances, there are also some bins with very high ratio, but as we can see in the top plots, these bins usually contain a very low number of events $(\sim 1-3)$. Their statistical significance is therefore low. The distribution of the differences between the lightning and magnetic footprints is not the same for the nighttime and daytime cases. The distribution is with a good approximation symmetric in daytime observations, whereas at nighttime, most of the lightning occur to the west of the magnetic footprint, which is probably a consequence of the fact that the lightning activity peaks at the local evening hours (Rycroft et al., 2000). We also have more lightning to the north of magnetic footprints. This is because the burst mode on DEMETER is usually switched off above Central Europe as the satellite "leaves" the seismically active zones. Thus, we have less burst mode VLF data when the satellite is north of thunderstorms detected by EUCLID. See also typical example in Fig. 3.

In Fig. 6 we show, how the mean whistler amplitude varies with the difference between the latitude and longitude of the magnetic footprint and causative lightning. As we mentioned previously, the distribution of lightning is rather asymmetrical with respect to the magnetic footprint in the case of nighttime observations; we will therefore limit our analysis to daytime observations here. The data are organized into bins with resolution of $1^{\circ}$ in latitude and longitude. Note that we don't

Fig. 5. Top: number of all lightning detected by the EUCLID network as a function of longitudinal and latitudinal difference between the magnetic footprint and locations of lightning. Bottom: Ratio of the assigned to all lightning (nighttime cases).

distinguish the lightning current here. Figure 6 demonstrates that the average amplitude of whistlers is largest when the magnetic footprint is approximately one degree to the north of the causative lightning location. This indicates an oblique propagation. Note that if the whistlers mostly propagated in ducts along the field lines, than the highest amplitudes would be observed for magnetic footprints just above the lightning locations. This is also consistent with the plane wave analysis and ray tracing study by Santolik et al. (2009), who found that the waves received on DEMETER entered the ionosphere $\sim 130 \mathrm{~km}$ equatorward from the magnetic footprint. We estimated the location of the highest amplitude in a map of mean whistler amplitudes as a location of centroid of mean whistler intensities calculated over the range $-5^{\circ}$ to $5^{\circ}$ of latitudinal and longitudinal differences, respectively, using Eq. (3).

$$
\left(x_{c}, y_{c}\right)=\left(\frac{\sum_{i j} I\left(x_{i}, y_{j}\right) x_{i}}{\sum_{i j} I\left(x_{i}, y_{j}\right)}, \frac{\sum_{i j} I\left(x_{i}, y_{j}\right) y_{j}}{\sum_{i j} I\left(x_{i}, y_{j}\right)}\right)
$$

$x_{c}, y_{c}$ is the position of the centroid, $x_{i}$ and $y_{j}$ are the values of latitudinal and longitudinal differences for a corresponding bin and $I\left(x_{i}, y_{j}\right)$ is the mean whistler intensity in the bin. We will call the point $\left(x_{c}, y_{c}\right)$ computed by Eq. (3) as reference point hereafter. Its location is $0.36^{\circ}$ of longitudinal difference and $1.15^{\circ}$ of latitudinal difference for daytime cases.

The difference between the magnetic footprint and the vertical projection of the satellite to the ground is $\sim 3-4^{\circ}$. It means that the reference point is closer to the magnetic 


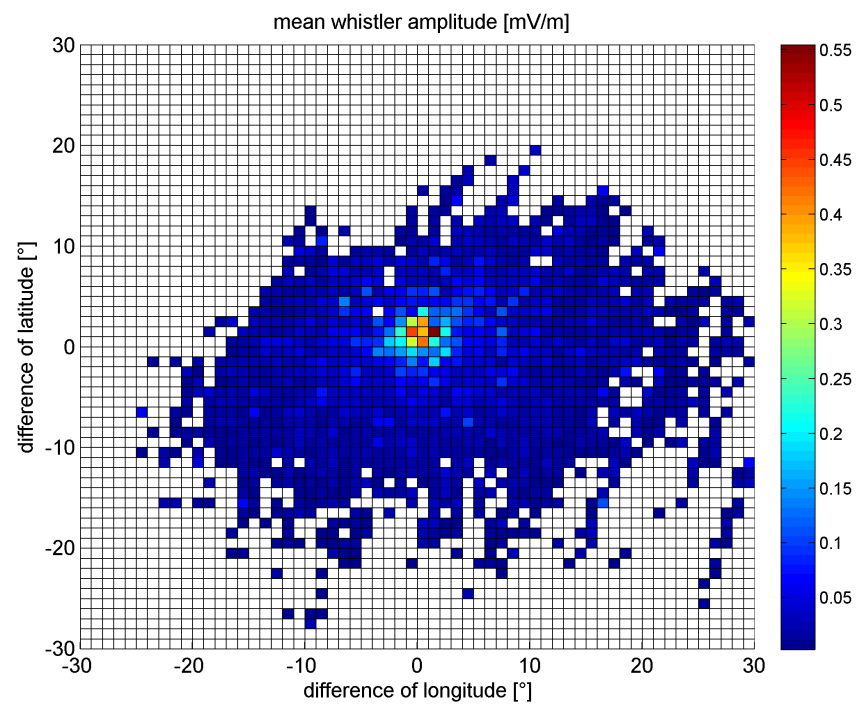

Fig. 6. Mean whistler amplitudes as a function of longitudinal and latitudinal difference between the magnetic footprint and locations of lightning for daytime cases.

footprint than to the vertical projection of DEMETER to the ground. This is not surprising because the propagation of whistler waves is controlled by the magnetic field even in a non-ducted case.

The average whistler amplitude as a function of discharge current and distance between the reference point and the causative lightning location for the daytime (late morning) cases is presented in Fig. 7. The data are again organized into bins, the step in the current is $10 \mathrm{kA}$ and the resolution in the distance is $100 \mathrm{~km}$. The bins which contained less than 20 events were discarded. The upper panels represent a map displaying the colour coded average whistler amplitudes in the individual bins. The bottom panels show whistler amplitude as a function of distance for lightning currents in the selected intervals $0-10 \mathrm{kA}, 10-20 \mathrm{kA}$ and 20 $30 \mathrm{kA}$. It is obvious that the whistler amplitude decreases with distance up to $\sim 1000 \mathrm{~km}$ and increases with the current of the causative lightning. For distances in the range $\sim 300-\sim 2000 \mathrm{~km}$, the decrease of mean whistler amplitude is, with a good approximation, inversely proportional to the distance; thus, the wave power decreases with the square of distance. That corresponds to the spherical spreading of that part of the energy that leaks into the ionosphere. Concerning the intensities in the Earth-ionosphere waveguide, we have no information. We only assume that their decrease is controlled by the net effect of quasi-cylindrical spreading and wave attenuation, which is expected to be different during the day and night. For distances larger than $\sim 1000 \mathrm{~km}$, the spreading of the wave is high and whistler amplitudes approach the detection limit. Thus, the width of the area of penetration is $\sim 2000 \mathrm{~km}$. The detection of whistlers at larger
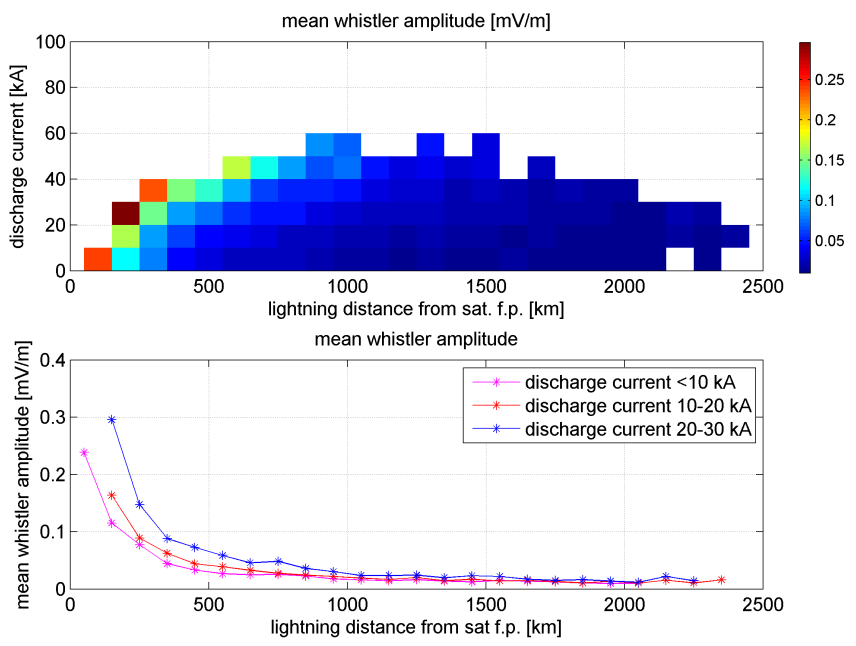

Fig. 7. Mean whistler amplitude as a function of lightning current and distance between the reference point and locations of lightning for daytime cases.

distances may also be limited owing to the limited area of the EUCLID detection network. The distance of $\sim 1000 \mathrm{~km}$ to which whistlers are detected is in agreement with the previous study (Chum et al., 2006). The highest amplitudes are usually observed up to distances of $\sim 500 \mathrm{~km}$. It should be also noted that we may missed weak whistlers in our detection algorithm. Thus, some undetected weak whistlers recorded on DEMETER can originate from distances larger than $\sim 1000 \mathrm{~km}$.

In Fig. 8 we show the mean whistler amplitudes as a function of distance between the reference point and the causative lightning location for the daytime cases separately for incloud and CG discharges. Organization of data is the same as in the bottom plot of the previous figure, except that we show only data for discharges with peak currents lower than $10 \mathrm{kA}$, because most of the in-cloud lightning used in our analysis have peak current in this range. There is no significant difference between whistler amplitudes for both types of lightning. A small difference is observed for distances less than $\sim 750 \mathrm{~km}$, which could probably be explained by different radiation patterns of CG and in-cloud discharges. The incloud discharges are often horizontal and thus, radiate more power in vertical direction. However, for distances less than $100 \mathrm{~km}$, we have observed a decrease of whistler amplitudes that correspond to the in-cloud discharges. First, it should be noted that for distances less than $100 \mathrm{~km}$, we have observed only few in-cloud discharges, thus the statistical significance of this point is relatively low - see also the vertical error bars showing the standard error of mean whistler amplitudes. Second and more important, we should stress that the distances are calculated to the reference point, which includes the effect of the trans-ionospheric propagation. This refrence point is not directly above the discharges that produce the most 


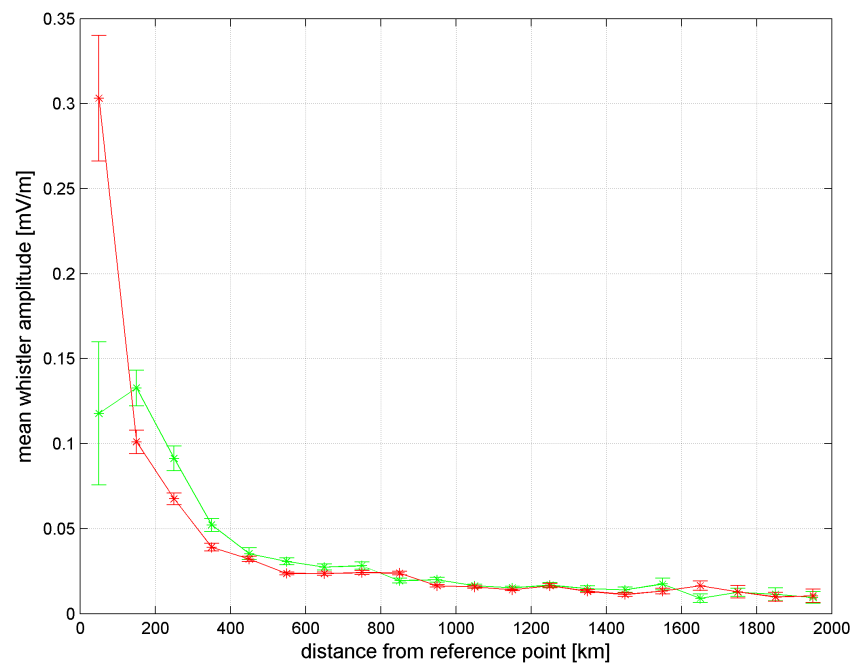

Fig. 8. Mean whistler amplitude as a function of distance between the reference point and locations of lightning for daytime cases. Green line - in-cloud discharges, red line - CG discharges. Error bars mark standart error of the arithmetic mean.

intense whistlers recorded by the satellite (see Eq. 3 and the related text). Thus, the curves presented in Fig. 8 represent the net effect of lightning radiation pattern and transionospheric propagation.

In Fig. 9, we show the whistler amplitudes as a function of lightning current for fixed distances between the reference point and lightning obtained from the maps representing the daytime and nighttime cases. The map for daytime cases is presented in Fig. 7. Different colours are used for different intervals of distances. Worth noticing is that the whistler amplitudes at nighttime are about 3 times larger than the amplitudes observed during the daytime. The lower amplitudes observed during the day can be explained by a relatively strong damping of the waves because of the collisions of electrons with neutral particles in the lower ionosphere in the $\mathrm{D}$ and/or E layer. At nighttime, these ionization layers are relatively weaker and shifted to higher altitudes where the collision frequencies are lower. From Fig. 9 it is obvious that the whistler amplitudes increase with the discharge current nonlinearly; the dependencies for fixed distances are roughly parabolic. However, the accuracy of these curves is relatively low. First, the average amplitudes are influenced by the limited number of data points in each bin and by the fact that the data were recorded during different satellite orbits. Though the satellite passes over the same region at about the same local time during the day and night, respectively, the ionospheric conditions can vary between the individual day passes and night passes, respectively, owing to different seasons and different solar and geomagnetic activity. Second, weak whistlers may not be detected due to the threshold used in the detection algorithm. This mainly influences the whistlers caused by
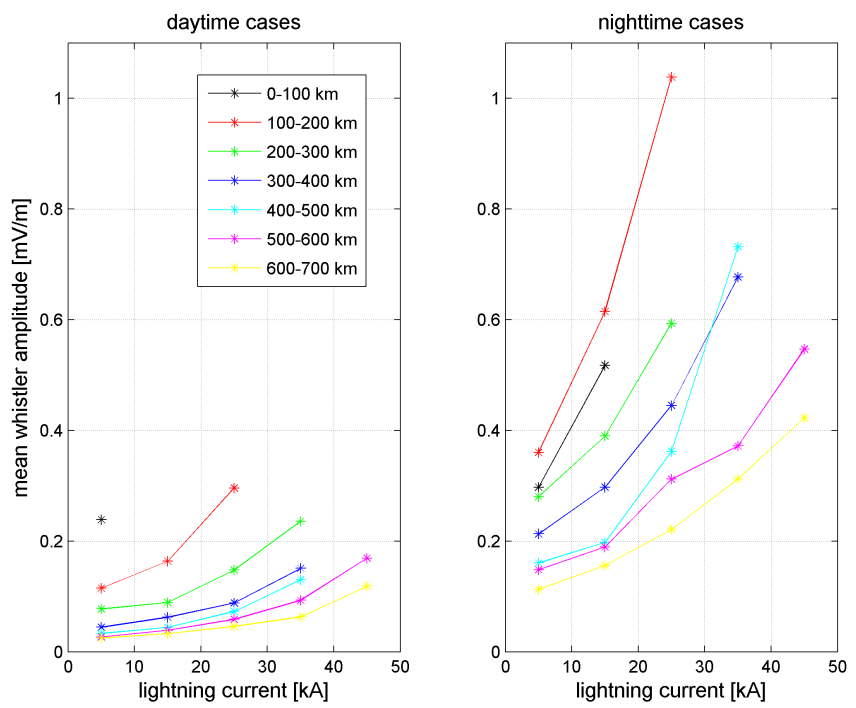

Fig. 9. Mean whistler amplitudes as a function of lightning current for fixed distances (colour-coded) between the reference point and locations of lightning. Left: Daytime cases, right: Nighttime cases.

low current discharges and/or whistlers detected at relatively large distances.

We should also mention that the amplitudes of the electric field component $E$ or the ratio $E / B$, respectively, depend on the plasma density which is generally different in the daytime and nighttime. That means, the measured electric field intensity can change during the day without any change of the wave damping and/or lightning intensities. To estimate the role of the plasma density on the electric field intensity we will assume for simplicity the collision-less plasma and unattenuated wave propagation. We will also assume that the propagation directions and the initial spectral intensities don't change during the day. Under these assumptions, the Poynting flux $S$ remains constant. The absolute value $S$ of $S$ averaged over one period can be expressed as follows (Eq. 9 in Chum et al., 2005):

$S=\frac{1}{2 \mu_{0}} \frac{k}{\omega} \sqrt{\rho_{X^{\prime} Z^{\prime}}^{\prime 2}+\left(1+\operatorname{Im}^{2}\left\{\rho_{X^{\prime} Y^{\prime}}^{\prime}\right\}\right)^{2}} E_{X^{\prime} 0}^{\prime 2}$

Where $E_{X^{\prime} 0}^{\prime}$ is the amplitude of the electric field in the plane defined by the magnetic field and $\boldsymbol{k}$ and having the direction perpendicular to $\boldsymbol{k}, \omega$ is the angular wave frequency and $\rho_{X^{\prime} Y^{\prime}}^{\prime}$ and $\rho_{X^{\prime} Z^{\prime}}^{\prime}$ are the polarization coefficients defined by Eqs. (5) and (6) in Chum et al. (2005). For small angles $\theta$ the the polarization coefficients $\rho_{X^{\prime} Y^{\prime}}^{\prime}$ and $\rho_{X^{\prime} Z^{\prime}}^{\prime}$ are close to $i\left(i^{2}=-1\right)$ and 0 , respectively. Thus, $E_{X^{\prime} 0}^{\prime}$ can be considered the amplitude of the electric field perpendicular to wave vector $\boldsymbol{k}$ and it is approximately measured by the ICE antenna. Using the simplified dispersion relation for 
quasi-longitudinal whistler mode waves $\left(\omega_{c} \cos \theta \gg \omega\right.$ and $\left.\omega_{p} \gg \omega\right)$ we obtain

$\frac{k c}{\omega}=\frac{\omega_{p}}{\sqrt{\omega \omega_{c} \cos \theta}}$

and substituting $k$ from Eqs. (5) into (4) we get for quasilongitudinal propagation

$$
S=\frac{1}{\mu_{0} c} \frac{\omega_{p}}{\sqrt{\omega \omega_{c} \cos \theta}} E_{X^{\prime} 0}^{\prime 2}
$$

Using Eq. (6) and assuming that the Poynting flux $S$ remains constant, we obtain the Eq. (7) which relates the electric field amplitudes $E_{\mathrm{X} 1}$ and $E_{\mathrm{X} 2}$ to two different plasma densities $n_{1}$ and $n_{2}$.

$$
E_{\mathrm{X} 1} / E_{\mathrm{X} 2}=\sqrt{\frac{\omega_{p 2}}{\omega_{p 1}}}=\sqrt[4]{\frac{n_{2}}{n_{1}}}
$$

where $E_{\mathrm{X} 1}$ corresponds to plasma density $n_{1}$ and $E_{\mathrm{X} 2}$ corresponds to plasma density $n_{2}$. Using the IRI 2007 model we found characteristic plasma densities $n_{1}$ and $n_{2}$ over the middle Europe $\left(50^{\circ} \mathrm{N}, 15^{\circ} \mathrm{E}\right)$ at altitude $660 \mathrm{~km}$ at 9 a.m. and 9 p.m., respectively. These times correspond approximately to the times of daytime and nighttime passes of DEMETER. We chose 6 days during the thunderstorm season from April to September. The calculations presented in Table 2 show that the ratio of electric field amplitudes $E_{\mathrm{X} 1} / E_{\mathrm{X} 2}$ corresponding to the diurnal variation of plasma density ranges from 0.89 to 1.03. However, the observed ratio of mean nighttime amplitudes to daytime amplitudes of whistler electric field is $\sim 3$ (see Fig. 9). Therefore, we conclude that the diurnal variations of plasma densities at the altitude of DEMETER cannot explain the observed diurnal differences of mean whistler amplitudes. We think that the diurnal variation of mean whistler amplitudes can be attributed to the variation of altitude of the base of the ionosphere. During the day, the ionosphere expands to lower altitudes (mainly D and E layers), than during the night. At lower altitudes, the collision frequencies between electrons and neutral particles are much higher, than at higher altitudes. This results to a stronger damping during the day, than during the night. The quantitative analysis is, however, beyond the scope of this experimental paper.

\section{Conclusions}

Three conclusions can be deduced from the obtained results: First, the amplitudes of whistlers decrease approximately inversely proportional to the distance between the magnetic footprint or reference point, respectively, and lightning (for distances larger than $\sim 300 \mathrm{~km}$ ). At distances larger than $\sim 1000 \mathrm{~km}$ the amplitudes approach the background noise. The highest amplitudes are usually observed up to distances of $\sim 500 \mathrm{~km}$.
Table 2. Characteristic plasma densities obtained from the IRI 2007 model at altitude $660 \mathrm{~km}$ over the middle Europe and corresponding ratios of the calculated electric field intensities (index 1 refers to daytime cases, 2 to nighttime cases).

\begin{tabular}{cccc}
\hline date & $n_{1}\left[10^{9} \mathrm{~m}^{-3}\right]$ & $n_{2}\left[10^{9} \mathrm{~m}^{-3}\right]$ & $E_{\mathrm{X} 1} / E_{\mathrm{X} 2}$ \\
\hline 1 Apr 2006 & 30.6 & 35.1 & 1.03 \\
1 May 2006 & 31.5 & 19.7 & 0.89 \\
1 Jun 2006 & 33.0 & 29.7 & 0.97 \\
1 Jul 2006 & 32.5 & 37.0 & 1.03 \\
1 Aug 2006 & 28.9 & 30.3 & 1.01 \\
1 Sep 2006 & 29.0 & 24.1 & 0.95 \\
\hline
\end{tabular}

Second, for fixed distances between the magnetic footprints and lightning, the average amplitudes of whistlers increase nonlinearly with the current of the causative lightning discharge.

Third, there is a significant difference between the mean whistler amplitudes observed during daytime and nighttime. The average amplitudes of whistlers at nighttime are about three times larger than the amplitudes observed during the daytime for the same range of lightning currents.

Acknowledgements. This work is based on observations with the electric field experiment ICE and the magnetic field experiment IMSC embarked on DEMETER launched by the Centre National d'Etudes Spatiales (CNES). We thank J. J. Berthelier for the DEMETER ICE electric field data. We thank all the member organizations of EUCLID for providing the lightning data. We would like to acknowledge the grant number 205/09/1253 of the Grant Agency of the Czech Republic. We also acknowledge valuable discussions with D. Skhlyar.

Topical Editor M. Pinnock thanks R. Marshall and A. R. W. Hughes for their help in evaluating this paper.

\section{References}

Abel, B. and Thorne, R.: Electron scattering loss in Earths inner magnetosphere1. Dominant physical processes, J. Geophys. Res., 103, 2385-2395, 1998a.

Abel, B. and Thorne, R.: Electron scattering loss in Earths inner magnetosphere 2. Sensitivity to model parameters, J. Geophys. Res., 103, 2385-2395, 1998b.

Barkhausen, H.: Whistling tones from the Earth, Proc. Inst. Radio Eng., 18, 1155-1159, 1930.

Berthelier, J. J., Godefroy, M., Leblanc, F., Malingre, M., Menvielle, M., Lagoutte, D., Brochot, J., Colin, F., Elie, F., Legendre, C., Zamora, P., Benoist, D., Chapuis, Y., Artru, J., and Pfaff, R.: ICE, the electric field experiment on DEMETER, Planet. Space Sci., 54, 456-471, 2006.

Betz, H. D., Schmitd, K., and Oettinger, W. P.: LINET - An international VLF/LF Lightning Detection Network in Europe, in: Lightning: Principles, Instruments and Applications, edited by: Betz, H. D., Schumann, U., and Laroche, P., chap. 5, pp. 115-140, Springer Science+Business Media B. V., doi:10.1007/ 978-1-4020-9079-0_5, 2009. 
Bortnik, J., Inan, U. S., and Bell, T. F.: Energy distribution and lifetime of magnetospherically reflecting whistlers in the plasmasphere, J. Geophys. Res., 108, 1199, doi:10.1029/2002JA09316, 2003.

Brinca, A. L.: On the stability of Obliquely Propagating Whistlers, J. Geophys. Res., 77, 3495-3507, 1972.

Chum, J., Jiricek, F., and Smilauer, J.: Nonducted propagation of chorus emissions and their observation, Planet. Space Sci., 53, 307-315, doi:10.1016/j.pss.2004.09.057, 2005.

Chum, J., Jiricek, F., Santolik, O., Parrot, M., Diendorfer, G., and Fiser, J.: Assigning the causative lightning to the whistlers observed on satellites, Ann. Geophys., 24, 2921-2929, 2006, http://www.ann-geophys.net/24/2921/2006/.

Crary, J. H., Helliwell, R. A., and Chase, R. F.: Stanford-Seattle whistler observations, J. Geophys. Res., 61, 35-44, 1956.

Cussac, T., Clair, M.-A., Ultré-Guerard, P., Buisson, F., LassalleBalier, G., Ledu, M., Elisabelar, C., Passot, X., and Rey, N.: The Demeter microsatellite and ground segment, Planet. Space Sci., 54, 413-427, doi:10.1016/j.pss.2005.10.013, 2006.

Eckersley, T. L.: Musical atmospherics, Nature, 135, 104-106, 1935.

Helliwell, R. A.: Whistlers and related ionospheric phenomena, Stanford University Press, 1965.

Holzworth, R. H., Winglee, R. M., Barnuma, B. H., and Li, Y.: Lightning whistler waves in the high-latitude magnetosphere, J. Geophys. Res., 104, 17369-17378, 1999.

Kennel, C. F. and Petschek, H. E.: Limit on stably trapped particle fluxes, J. Geophys. Res., 71, 1-28, 1966.

Kimura, I.: Effects of ions on whistler-mode ray tracing, Radio Sci., 1, 3, 269-283, 1966.
Lagoutte, D., Brochot, J. Y., de Carvalho, D., Elie, F., Harivelo, F., Hobara, Y., Madrias, L., Parrot, M., Pinçon, J. L., Berthelier, J. J., Peschard, D., Seran, E., Gangloff, M., Sauvaud, J. A., Lebreton, J. P., Stverak, S., Travnicek, P., Grygorczuk, J., Slominski, J., Wronowski, R., Barbier, S., Bernard, P., Gaboriaud, A., and Wallut, J. M.: The DEMETER Science Mission Centre, Planet. Space Sci., 54, 428-440, doi:10.1016/j.pss.2005.10.014, 2006.

Prentice, S. A. and Mackerras, D.: Ratio of cloud to cloud-ground lightning flashes in thunderstorms, J. Appl. Meteor., 16, 545550, 1977.

Rycroft, M. J., Israelsson, S., and Price, C.: The global atmospheric electric circuit, solar activity and climate change, J. Atmos. Solar-Terr. Phys., 62, 1563-1576, doi:10.1016/S1364-6826(00) 00112-7, 2000.

Santolik, O., Parrot, M., Inan, U. S., Buresova, D., Gurnett, D. A., and Chum, J.: Propagation of unducted whistlers from their source lightning: A case study, J. Geophys. Res. (Space Physics), 114, 3212, doi:10.1029/2008JA013776, 2009.

Shklyar, D. R., Chum, J., and Jiř́ícek, F.: Characteristic properties of $\mathrm{Nu}$ whistlers as inferred from observations and numerical modelling, Ann. Geophys., 22, 3589-3606, 2004, http://www.ann-geophys.net/22/3589/2004/.

Smith, R. L. and Angerami, J. J.: Magnetospheric properties deduced from OGO 1 observations of ducted and nonducted whistlers, J. Geophys. Res., 73, 1-20, 1968.

Storey, L. R. O.: An investigation of whistling atmospherics, Phil. Trans. Roy. Soc. London, 246, 113-141, 1953.

Trakhtengerts, V. Y., Rycroft, M. J., Nunn, D., and Demekhov, A. G.: Cyclotron acceleration of radiation belt electrons by whistlers, J. Geophys. Res., 108, 1138, doi:10.1029/ 2002JA009559, 2003. 